\title{
NOTE
}

This is a draft only, the final published version of this paper is available in the March 2014 edition of CIN: Computers, Informatics, Nursing: (March 2014 - Volume 32 Issue 3 - p 101-104)

doi: 10.1097/CIN.0000000000000049

http://journals.lww.com/cinjournal/Citation/2014/03000/Implications_for_Research_M ethods_When_Conducting.1.aspx

\section{Implications for research methods when conducting studies with the users of online health communities}

\author{
Carol S Bond ${ }^{1}$, Osman Hassan Ahmed ${ }^{1}$, Martin Hind ${ }^{1}$.
}

1. School of Health and Social Care, Bournemouth University, Bournemouth, England.

\section{Corresponding author:}

Carol S Bond, The School of Health and Social Care, Bournemouth University, Royal London House, Christchurch Road, Bournemouth, Dorset, BH1 3LT, England.

Key words: $\quad$ Diabetes; Internet; Research Ethics; Self-Help Groups.

- Online health communities are a valuable and established source of information and peer support for people living with a long term condition

- Including these online community participants in research brings methodological considerations

- Established research norms need to be applied pragmatically to ensure research is carried out efficiently and ethically. 


\section{Introduction}

Online communities are present for almost every health condition, with these online spaces allowing people to connect with each other ${ }^{1}$. Increasingly, health researchers are exploring these communities in order to study the rich information contained on these websites and to observe how people are using these communities ${ }^{2-4}$. This article was created as a consequence of the research team's experiences with conducting email interviews with users of online diabetes communities. A fuller report of the research and findings is available ${ }^{5}$, whilst this paper explores the challenges and successes that we experienced as researchers.

The original research sought the views of contributors to online diabetes discussion boards about how they felt their posts on these boards should be used by health researchers. The recruitment and interviews were undertaken entirely online. Although the research team had prior experience with researching online communities ${ }^{6,7}$, this particular project threw up considerations which were felt to be worthy of discussion and dialogue with a wider health research community.

\section{Challenges in conducting this form of research and lessons learned}

During the course of our research, methodological considerations arose associated with appropriate engagement with participants online. In addition, practical issues with conducting this research efficiently and ethically are also discussed. The key challenges that we encountered during this research are summarised below, followed by factors to consider for researchers looking at exploring online health discussion forums.

\section{Accessing Online Community Participants}

The communities sampled for our study had a mixture of registration requirements. All of the discussion boards were public, in that they allowed all posts to be read without any registration. For some boards however the rules stated that it was necessary to register and create a username and password in order to be able to post to the board. This process did not require people to say why they wanted to register with the site, or to declare any connection with the health condition. We therefore did not feel we were breeching any trust or expectations for board membership and registered to gain access for posting if necessary. During the registration process we did have to agree to abide by the board rules and/or terms and conditions. This necessitated reading the small print to ensure that researchers were not excluded. During the course of participant recruitment it was important for the research team 
to be open and honest about the reasons for their membership of the community, and not to pretend to be a person living with diabetes or mislead others in any way.

\section{Completeness of interviews}

We asked our participants 6 interview questions, spread across 4 emails. We aimed to send the next email as soon as practical after receiving the reply to the previous one. Most people completed the interviews in a few days. We expected to see variances in the sizes of answers in email interviews; whatever the interview format some participants will always have more to say than others. Some of our respondents elected to answer questions in several words, whilst others expanded over several pages. During the process of data collection we did not push or seek to persuade participants to add to short responses, as we were keen not to put them under any pressure or to put them off continuing. The converse of this however is that we may have missed an opportunity to obtain richer information by failing to do so.

The norm is to include verbatim quotes in the reporting of results in qualitative research. When you record interviews, it is generally considered good practice to include a participant ID or reference in order to ensure that the quotes are not restricted to a core few individuals and that your quote is used correctly. When faced with contributions covering quite a large range of different sizes as in our research, we found this principle to be even more important.

Whilst conducting online interviews, it can be anticipated that there will be at least a few interviews which remain uncompleted. Just as participants have the right to withdraw from a face-to-face interview at any time, they can also cease participation in an online interview. If an individual has already provided answers to some interview questions but fails to answer the remainder of the questions then it raises the issue of what to do with the incomplete interview data. In qualitative research the one of the principles in deciding how many interviews to carry out is the concept of reaching data saturation; the point at which further interviews produce no new categories, or themes ${ }^{8}$. In our research saturation of data was achieved via the completed interviews, however researchers should consider (and make arrangements for) how to deal with incomplete interview data, especially if it contains new information. Our view was that had we not reached saturation we would have included the results of incomplete interviews in our data analysis, as long as participants had not made any suggestion that they wished to withdraw. 


\section{Sampling}

Collecting demographic details such as age, sex and location assists the reporting of results, and it was our intention to collect this information from participants in this research. Our initial research plan aimed to use a purposive sampling approach, identifying people based on age and gender in order to provide a balanced interview sample. A preliminary review of online communities showed that people often included this type of information in their profile. However when we came to the data collection phase of our research not enough people who had included demographic information in their profiles were able to be contacted directly, and therefore the opportunities for purposive sampling were limited, and we could not sample in this way. Instead we had to post requests for participants on the boards, and wait for people to approach us.

Although we could have asked about demographic information to aid the presentation of our results we decided not to as this was background information and not essential to meet the research aims. Depending on your circumstances, you will have to make your own decisions about how to handle this issue. As a consequence, gender was the only demographic that was reported in our results. Although many of the participants had usernames from which their gender could be inferred, some participants had pseudonyms which were ambiguous and did not permit the identification of gender. In these instances where the gender was not able to be clearly identified, it was reported as "not possible to classify".

\section{Differences between communities}

In our research we found that no two online communities are identical. Although communities may have a focus towards the same pathology/condition, they can operate entirely differently, promote different advice and information, and have a totally different composition of people who use the group. Our observations showed the varying nature of communities to be apparent; one of the communities examined stated that they were a closeknit group with some of the members meeting face-to-face several times a year, whilst others had a wider membership base which would not permit this. This has implications when attempting to aggregate information collected from a variety of online communities.

In analysing the data from these communities, one of the challenges exists in identifying the culture of each community, because this will help to shape comparisons between the views and opinions between the communities. In addition, the difference in functionality between 
communities means that varying amounts of information is visible which has consequences when attempting to collect details on participants. Some of the discussion boards on the communities included in our research showed information regarding the number of posts a user had made or the date a user had joined the community, which could suggest a level of association an individual had with a community. Comparisons could not be made between all of the participants in this research however, as not all of the communities displayed this information.

\section{Ethical considerations}

Satisfying ethical review committees prior to conducting research with online health communities is a process which requires the adoption of general ethical principles consistent to all forms of research, for example the right of participants to withdraw from the research at any time. Research using online health communities also creates additional considerations which are specific to the online environment (e.g. how the research team communicate with participants), and these issues need to be carefully thought out prior to the research commencing in order to safeguard the well-being of the participants.

Recruitment of participants is always a challenge in all forms of research and must be done in an ethical manner. When we were not able to directly contact the owners of the discussion boards of the communities in this research, we identified the "gatekeeper", e.g. the discussion board moderator for recruitment. Where possible, we sought their consent prior to attempting to recruit from each community. We obtained participant permission from an individual's response to the request for participants posted on each of the forums. Although the use of online consent forms was considered, we agreed that this process would have resulted in people sharing a degree of personal information with the research team (e.g. their full name). Given that every attempt was made to preserve the anonymity of the participants, the use of online consent forms was not adopted. We did however ensure that each participant received a full information sheet explaining the research before the interviews commenced.

\section{Encouraging aspects for other researchers}

We are sharing our approaches and our experiences here because overall we found they were successful. Some of the positive experiences included the following: 


\section{Desire to participate}

After participant recruitment had ended, people continued to express an interest in participating in the research and the research team had to turn participants away. This reflected the fact that many participants appeared genuinely pleased to help with the research, and the verbatim quotes from participants reinforced this.

\section{Users recruiting other users}

Participants aided the recruitment process of the research by posting on the discussion boards within these online communities to share their positive experiences of participating in our research, and requesting that other users participate too. These posts were unprompted by the research team and indicated a willingness and desire from users to support research within the community.

\section{Low rate of attrition}

There was a relatively low rate of attrition compared to other internet studies. Of the 33 people who initially expressed an interest in participation, 30 people consented to participate and 26 completed their interviews.

\section{Other recommendations for researchers}

Prior to conducting our research, consideration was given as to the most effective method of collecting data from these interviews. Many online health communities have a direct messaging (DM) facility which allows instant responses and the rapid collection of data in this manner was an appealing aspect. However for the purposes of this research an asynchronous interview technique was utilized in order to allow participants time to reflect on their answers ${ }^{9}$ and thus email was the preferred method of communication. Researchers intending to communicate with people in online communities should think through what they wish to achieve from their research in order to carefully select the optimal method of data collection.

An issue which was implied by participants, and also directly mentioned during recruiting via the forums, was that discussion boards on online health communities receive many requests for assistance with research every week. This being the case, why should users of a community help with your research? It is important to be honest and upfront during this process in order to establish trust between your research team and the community. Several 
people who contacted the research team were initially suspicious about the intentions of our research as a result of negative previous experiences with others researchers who had contacted their community. Poorly designed studies, especially by students learning about research, were often criticised by members of these communities. We used our University contact details and shared our clinical and academic backgrounds as part of establishing our credibility. By clearly explaining the aims of our research, people who were initially suspicious were happy to take part in the project and contributed valuable data. It is important for those intending to study online health communities to be clear and unambiguous from the outset with regards to this.

A further point to consider is how will your research aid or assist the users of the community? Time is a precious commodity in modern society, and people will not want to spend valuable minutes or even hours taking part in a study if they can see no tangible benefit (either directly or indirectly) to themselves or to their community. By explaining how your research will aid their community and the "greater good" which will arise from participation, you will find that people are more likely to engage with the project. The results of our research have been published ${ }^{5}$, a short summary version was produced and posted on the boards and people were told how and where they could find the full published results. These steps were well-received by participants, and proactive steps of engagement such as this are valuable in building trust between researchers and participants. Such measures also aid the dissemination of findings to the primary stakeholder group, namely the users of these communities.

\section{References}

1. Eysenbach G, Powell J, Englesakis M, Rizo C, Stern A. Health related virtual communities and electronic support groups: Systematic review of the effects of online peer to peer interactions. British Medical Journal. 2004;328(7449):1166.

2. Letourneau N, Stewart M, Masuda JR, et al. Impact of online support for youth with asthma and allergies: Pilot study. Journal of Pediatric Nursing. 2012;27(1):65-73.

3. Gold KJ, Boggs ME, Mugisha E, Palladino CL. Internet message boards for pregnancy loss: Who's on-line and why? Women's Health Issues. 2012;22(1):e67-e72.

4. Gilbert K. Online communities are valued by people with type 1 diabetes for peer support: How well do health professionals understand this? Diabetes spectrum. 2012;25(3):180-191.

5. Bond CS, Ahmed OH, Hind M, Thomas B, Hewitt-Taylor J. The Conceptual and Practical Ethical Dilemmas of Using Health Discussion Board Posts as Research Data. J Med Internet Res 2013;15(6):e112 http://www.jmir.org/2013/6/e112/. 2013. 
6. Bond C. Researching online: virtually the same? 5th Qualitative Research Conference in Health and Social Care; 2004; Bournemouth University, Bournemouth, UK.

7. Ahmed OH, Sullivan SJ, Schneiders AG, McCrory P. iSupport: Do social networking sites have a role to play in concussion awareness? Disability and Rehabilitation. 2010;32(22):18771883.

8. Guest G, Bunce A, Johnson L. How Many Interviews Are Enough? Field Methods. 2006;18(1):59-82.

9. James N, Busher H. Online Interviewing. Sage; 2009. 